\title{
Rhythm Comes, Rhythm Goes: Short-Term Periodicity of Prosodic Phrasing
}

\author{
Sabrina Stehwien ${ }^{1}$ and Lars Meyer ${ }^{1,2, *}$ \\ ${ }^{1}$ Max Planck Research Group Language Cycles \\ MPI for Human Cognitive and Brain Sciences, Leipzig, DE \\ ${ }^{2}$ Clinic for Phoniatrics and Pedaudiology \\ University Hospital Münster, DE
}

March 10, 2021

${ }^{*}$ Corresponding author-lmeyer@cbs.mpg.de 


\section{Abstract}

Speech is perceived as a sequence of meaningful units. Speech prosody helps to delimit these units through pauses and acoustic modulations of pitch, amplitude and speech rate. These prosodic boundaries subdivide utterances into prosodic phrases. To be understood, prosodic phrases must obey cognitive and neurobiological constraints on the side of the listener. In particular, the neurobiological substrates of speech processing have been argued to operate periodically — with one electrophysiological processing cycle being devoted to the processing of one segment of the speech stream. We hypothesized that when processing is periodic, prosodic phrases should show periodicity as well. We investigated the periodicity of prosodic phrases in a corpus of radio news that has been manually annotated for full intonational and intermediate phrases by human experts. We find that sequences of 2 to 5 intermediate phrases are periodic at 0.8 to 1.6 Hertz within their superordinate intonation phrase. Across utterances, the exact duration of intermediate phrases fluctuates with the duration of superordinate intonation phrases, pointing to a dependence of prosodic time scales. Our findings provide evidence of short-term periodicity of prosodic phrasing within a highly specific range. While the determinants of periodicity are unknown, the results are compatible with an association between electrophysiological processing time scales and the phonological rhythms of language as such. This is a further step towards closing the gaps between the neurobiology of language, psycholinguistics, and linguistic description. 


\section{Introduction}

Behind speech, there is a hierarchical structure of phonological units - phonemes form syllables, syllables form words, and so on. At the topmost level, there are patterns of rhythm, stress, and tune, referred to as prosody (Selkirk, 1984; Ladd, 2008; Nespor \& Vogel, 2007). Apart from paralinguistic meaning such as emotion and arousal, prosody conveys the phrasing of an utterance, that is, the succession of multi-word units termed intonation phrases (Ladd, 2008; for review, see Féry, 2017).

There is no universally accepted definition of intonation phrases, partly due to substantial cross-linguistic variability (Jun, 2010). It is generally agreed upon that they are delineated by prosodic phrase boundaries - multi-dimensional combinations of a falling or rising pitch contour, often followed by a pause and a pitch and energy reset (for an overview, see Brugos et al., 2018). The widely-used Tones and Break Indices (ToBI) annotation system further distinguishes between different types that are organized hierarchically: Longer and more distinct intonation phrases (IPs) consist of shorter intermediate phrases (ips). IP boundaries are perceived as more salient than ip boundaries; furthermore, ip boundaries are often not marked by pauses. In ToBI, ip and IP boundaries correspond to the two strongest break indices 3 and 4, respectively (Silverman et al., 1992; Pierrehumbert, 1980).

IPs are a critical object of psycholinguistics (Frazier et al., 2006). Prosodic phrasing guides the cognitive formation of macroscopic syntactic and semantic units (Kreiner \& Eviatar, 2014; Wagner \& Watson, 2010; Cutler et al., 1997). IPs roughly correspond to syntactic units, whereby prosodic boundaries scale with the size of the unit (Grosjean et al., 1979; Truckenbrodt, 1999). In general, listeners employ prosodic boundaries to segment verbal stimuli (Bower \& Springston, 1970; Farrell \& Lelièvre, 2012; Sturges \& Martin, 1974; Ghitza, 2017); in particular, behavioral and electrophysiological data indicate that prosodic boundaries trigger the termination of syntactic units (Lehiste et al., 1976; Price et al., 2015; Wightman et al., 1992; Snedeker \& Casserly, 2010; Steinhauer et al., 1999). It is often proposed that the sampling of the words contained within an IP allows the listener to decode a single coherent piece of information (Inbar et al., 2020; Wahl, 2015; Speer \& Ito, 2009).

Why is speech subdivided into prosodic units, rather than providing an uninterrupted stream of information? Working memory limitations may be a cognitive reason (Chafe, 1979; Simpson \& del Prado Martín, 2015; Speer et al., 1993): The multiple words of a prosodic phrase do not occur simultaneously. Hence, the decoding of the information contained within a prosodic phrase must finish before phrase-initial words are forgotten (Christiansen \& Chater, 2015). Information held in working memory deteriorates within few seconds (Wingfield \& Byrnes, 1972; White, 2017). Behavioral and electrophysiological research suggests that once this limit is approached, macroscopic syntactic units 
are terminated regardless of the presence or absence of prosodic boundaries (Hwang \& Steinhauer, 2011; Roll et al., 2012; Webman-Shafran et al., 2015).

Recent neuroscientific research suggests that instead of - or in parallel to - cognitive constraints, neuronal constraints may limit prosodic phrase duration; in particular, the temporal regularity of electrophysiological information processing. Neural oscillations are fluctuations in the electrical potentials of neurons and neuronal ensembles, which are involved in sensory sampling of external information as well as internal information exchange across brain networks (Buzsáki, 2006, 2019). Critically, it has been shown that auditory sampling proceeds in a rhythmic fashion, where time windows are in lockstep with oscillatory cycles (Hickok et al., 2015; Henry \& Obleser, 2012; Schroeder \& Lakatos, 2009). During speech processing in particular, oscillations in the auditory cortices synchronize with acoustic rhythms on the time scales of phonemes, syllables, and prosody (for review, see Giraud \& Poeppel, 2012; Meyer, 2018). It has been shown that acoustic amplitude modulations that are present in speech can mechanistically drive oscillations into synchronicity (Szymanski et al., 2011).

A periodic electrophysiological mechanism for the processing of prosodic phrases would entail that these are not merely limited in duration, but that their duration is regular in time. Temporal regularity of prosody is a recent hypothesis in linguistics (Gibbon, 2018) as well as a frequent assumption in neuroscience. To date, the empirical support is limited (Cummins, 2012; Goswami \& Leong, 2013; Kelso et al., 1986). The quantification of rhythmicity in speech corpora is an emerging field of linguistic research (Gibbon, 2018; Tilsen \& Arvaniti, 2013; Arvaniti, 2012). Initial support suggests that syllable frequency across languages is confined to a narrow band between 4 and 8 Hertz (Ding et al., 2017; Coupé et al., 2019; Tilsen \& Arvaniti, 2013), consistent with the previously proposed role of theta-band synchronization in syllable processing (Luo \& Poeppel, 2007; Peelle et al., 2013; Doelling et al., 2014; Howard \& Poeppel, 2012). Above the syllabic rate, a recent study found that the onset of intonation units that are analogous to ToBI's ip (Chafe, 1979; Du Bois, 1992) associates with a consistent phase angle of the speech envelope at a frequency of $\sim 1$ Hertz (Inbar et al., 2020). This suggests that amplitude modulations at prosodic boundaries may indeed be sufficiently regular for synchronizing a periodic neuronal sampling mechanism.

In this report, we do not investigate amplitude modulations as such. Instead, we make use of a corpus of German radio news that has been manually annotated with ToBI prosodic labels (Eckart et al., 2012), allowing us to investigate periodicity of prosodic phrase boundaries that take into account the multiple acoustic dimensions that trigger human perception (Brugos et al., 2018). We analyse the durations of both long IPs and the subordinate short ips. We find robust evidence of short-term periodicity at the level of prosodic phrasing. In more that 60 percent of superordinate IPs, we observed periodic series of 2 to 5 subsequent subordinate ips. Furthermore, we present evidence 
that periodicity is not fixed to one particular frequency, but ranges within a narrow band from roughly 0.8 to 1.6 Hertz, critically depending on the duration of the superordinate IP. We discuss implications for phonological theory, the neurobiology of language, and the interplay between neurobiological constraints and speech as such.

\section{Data acquisition}

The DIRNDL corpus is a database of radio news broadcasts collected from a German radio station (Eckart et al., 2012). The corpus was designed for linguistic analyses and annotated for phonological, syntactic, and information status information. We used the second release of the corpus (Björkelund et al., 2014). The prosodically labelled subset comprises around 5 hours of speech from several different professional speakers (male and female). The transcriptions are time-aligned to the speech signal. Since the data was collected over the course of a few days, the news items are repeated, but not always read by the same speaker or with the same prosody. The prosodic annotations follow the guidelines for the Stuttgart German adaptation of the ToBI system (GToBI(S); Mayer, 1995). In ToBI, IPs are marked by intonational phrase boundaries and ips are marked by intermediate phrase boundaries.

\section{Data analysis}

Data was pre-processed using Python; statistical analyses were performed in $\mathrm{R}$ ( $\mathrm{R}$ Core Team, 2017). The corpus contains 3,947 IPs and 9,041 ips. As initial step to assessing the regularity of IP and ip durations, we calculated durations and variances (see Results). We also explored the relationship between the durations of IPs and ips, based on the visual impression of a harmonic relationship; no outliers were removed for this analysis (Figure 1C). To test for an increase of ip duration with IP duration, we used linear mixed-effects model comparison implemented in the lme4 package (Bates et al., 2015). The dependent measure was ip duration. The baseline model included random intercepts to account for speaker- and recording-specific differences. Through Analysis of Variance (ANOVA), the random-effects-only model was compared to an improved model that added IP duration as a fixed effect (Barr et al., 2013).

Based on the results (see Results), we analyzed periodicity of ips within IP duration bins of 1 second width; due to the lack of long IPs, we considered IPs of up to 9 seconds. As an initial step, we compared the variance across ip durations within any IP that consists of at least two ips (total number of IPs $=2,664$; average number of ips per IP $=2.87$ ) to the variance of ip durations across the corpus (see Results). While this indicated regularity of ips within IPs, visual inspection did not show equispacing. Instead, the first ip appears longer than subsequent ips, which are again longer than the final ips (Figure 2A). To test 
this, we added the linear ip index as additional fixed effect and tested for improvement over the model that included IP duration as the only fixed effect. Because this led to improvement (see Results), we reasoned that ip duration could be less variable across immediate neighbors as compared to distant ips. To assess this, we calculated duration variance across pairs of increasing distance within IP duration bin (i.e., ips 1-2, 2-3, etc. versus ips $1-3,2-4$, etc. versus ips $1-4,2-5$, etc.; see Table 1 )

To provide direct evidence for short-term periodicity of entire sequences instead of pairs of ips, we then ran autocorrelation analyses on series of successive ips within IP duration bin. Because the previous analysis hinted at a decrease in ip duration variance with decreasing ip distance, we ran separate analyses for ip sequences of incrementally increasing ip count. Each ip series was converted into a binary time series, where 1 marks an ip offset. A sampling rate of 100 Hertz was chosen, that is, each one or zero marks a 10-millisecond frame. To avoid aliasing, the maximum lag matched the first occurrence of an ip offset. To determine significant lags, we performed a permutation test within individual ip series. We created 1,000 permutations of each series to obtain 1,000 random autocorrelation functions. A lag was considered significant if the observed $R$ was above the $97.5^{\text {th }}$ percentile of the random autocorrelations at this lag (corresponding to $p<0.05$, corrected; Maris \& Oostenveld, 2007). We removed all ip series for which the only significant lag was the first lag; an according period of $10 \mathrm{~ms}$ would likely not indicate meaningful periodicity. Due to the lack of long ip series, the maximum ip count was 7 .

Visual inspection suggested that significant lags were normally distributed for shorter ip series, whereas an increasing ip count associates with non-normal histograms (Figure 2B). To test this, we calculated, within duration bin and ip count, the Kullback-Leibler Divergence (KLD) between the distribution of lags and a normal distribution. A small $K L D$ would indicate a consistent period of ip offsets. The baseline normal distribution consisted of 10,000 random data points organized into 100 bins, based on the mean and standard deviation of the lag distribution. We bootstrapped a median $K L D$ value from 10,000 iterations of this procedure (Figure 3A). Median KLD values were then correlated with ip count. To account for covariance between ip count and IP duration bin (i.e., large ip counts are only present in long IPs), we partialed out IP duration bin. Because of the skewness of the $K L D$ distribution, we employed Kendall's partial rank correlation.

\section{Results}

IP duration across the corpus is $3.8 \mathrm{~s}$ (Figure 1A); variance is $3.53 \mathrm{~s}^{2}$. Some few IPs are very long (up to over 20 seconds), likely as a result of human annotator errors. After outlier removal according to the interquartile criterion, the variance drops to $2.6 s^{2}$. Median ip duration across the corpus was $1.65 \mathrm{~s}$; the first and third quartiles were at 1.15 and $2.29 \mathrm{~s}$, respectively (Figure 1B). The total variance is $0.69 s^{2}$, and $0.63 s^{2}$ after outlier removal. 

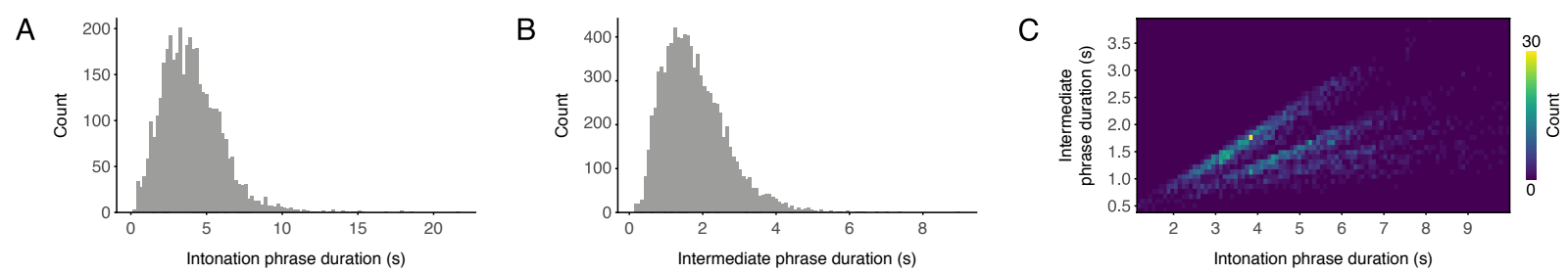

Figure 1: Distribution of IP/ip durations. (A) Histogram of IP durations. (B) Histogram of ip duration. (C) Two-dimensional histogram of ip duration (ordinate) as a function of IP duration (abscissa); colors indicate counts within $100 \times 40$ equispaced bins.

\begin{tabular}{llcccccccccc}
\hline & \multicolumn{10}{c}{ Distance between ips } \\
\cline { 2 - 12 } Measure & 1 & 2 & 3 & 4 & 5 & 6 & 7 & 8 & 9 & 10 & 11 \\
\hline Pairs (count) & 4,975 & 2,311 & 892 & 349 & 141 & 63 & 32 & 14 & 5 & 2 & 0 \\
Variance $\left(s^{2}\right)$ & 1.23 & 1.13 & 1.20 & 1.46 & 1.30 & 1.53 & 1.73 & 0.75 & 0.15 & - & \\
\hline
\end{tabular}

Table 1: Variance of pairwise differences in intermediate phrase length with increasing distance between each pair of phrases (counted in number of intermediate phrases). Distance of one means that the phrases are neighbors.

Maximum ip duration is $9.02 \mathrm{~s}$; hence, ips are shorter and less varied than IPs.

The joint distributions of IP and ip (Figure 1C) indicated an increase in ip duration with IP duration. In addition, there appeared to be two to three clusters of preferred ip durations : For IPs of $3 \mathrm{~s}$, the plot suggests ip durations of roughly 1.0 and $1.5 \mathrm{~s}$, for IPs of $4 \mathrm{~s}$, durations of 1.2 and almost $2.0 \mathrm{~s}$, and for IPs of $5 \mathrm{~s}$ a durations of 1.5 and $2.4 \mathrm{~s}$. The clusters spread with IP duration, with the higher-mean cluster as a multiple of around 1.5 to 1.6 of the smaller-mean cluster, roughly matching the median duration of an ip. Mixed-effects model comparison found the fixed effect of IP duration on ip duration to improve model fit over the random-effects-only baseline $\left(\chi^{2}(1)=301.8, p<0.001\right)$. This indicates that that IP duration explains ip duration with statistical significance.

The average variance of ips within IP is $0.39 s^{2}\left(0.28 s^{2}\right.$ after outlier removal), that is, smaller than the overall variance of ips in the corpus. While this is a first indication that ips are regular in time, the inclusion of the ip position within IP as a fixed effect led to a further improvement in model fit $\left(\chi^{2}(1)=430.54, p<0.001\right)$. Together, this suggests that ip duration is regular, but not constant. In line with this, ip duration variance was lower for nearby ips (Table 1). Together, ip periodicity is thus more likely to be found within shorter stretches of speech.

Autocorrelation analyses yielded a significant lag in 4,474 out of 7,346 ip series (i.e., $61 \%$ of ip series), pointing to substantial periodicity of ip series within IPs. The correlation between $K L D$ and ip count, partialing out IP duration bin, was significant $\left(\mathrm{r}_{\tau}=0.62\right.$, $\mathrm{p}$ $<0.001$; Figure 3B). This indicates that periodicity was high within short series of 2 to 5 ips, providing direct evidence for short-term periodicity of ips. 
A

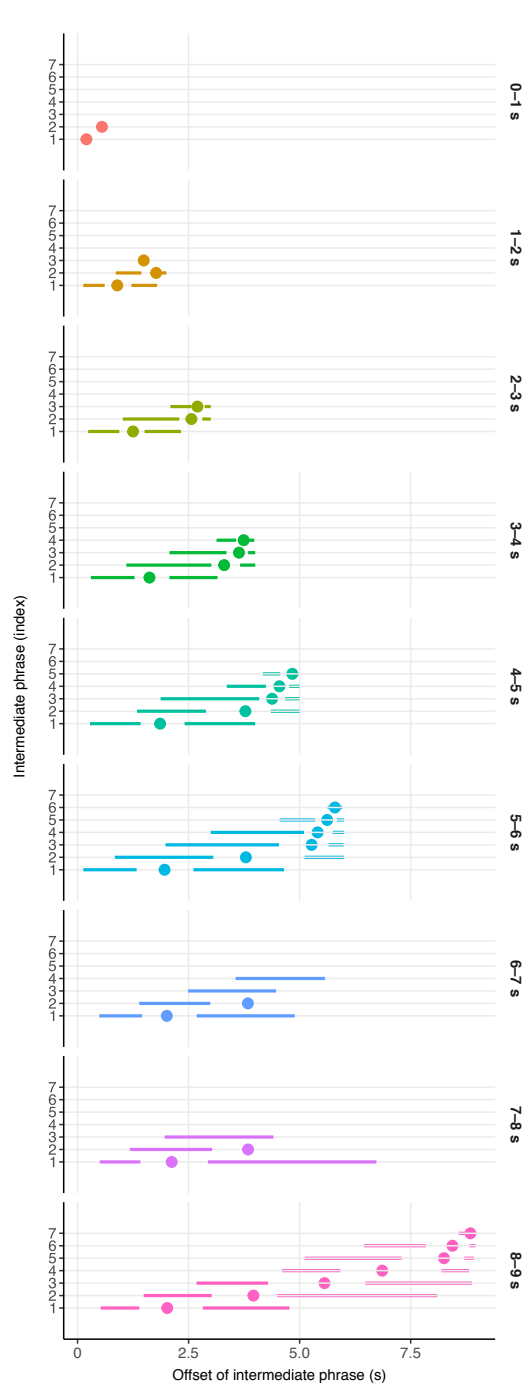

B

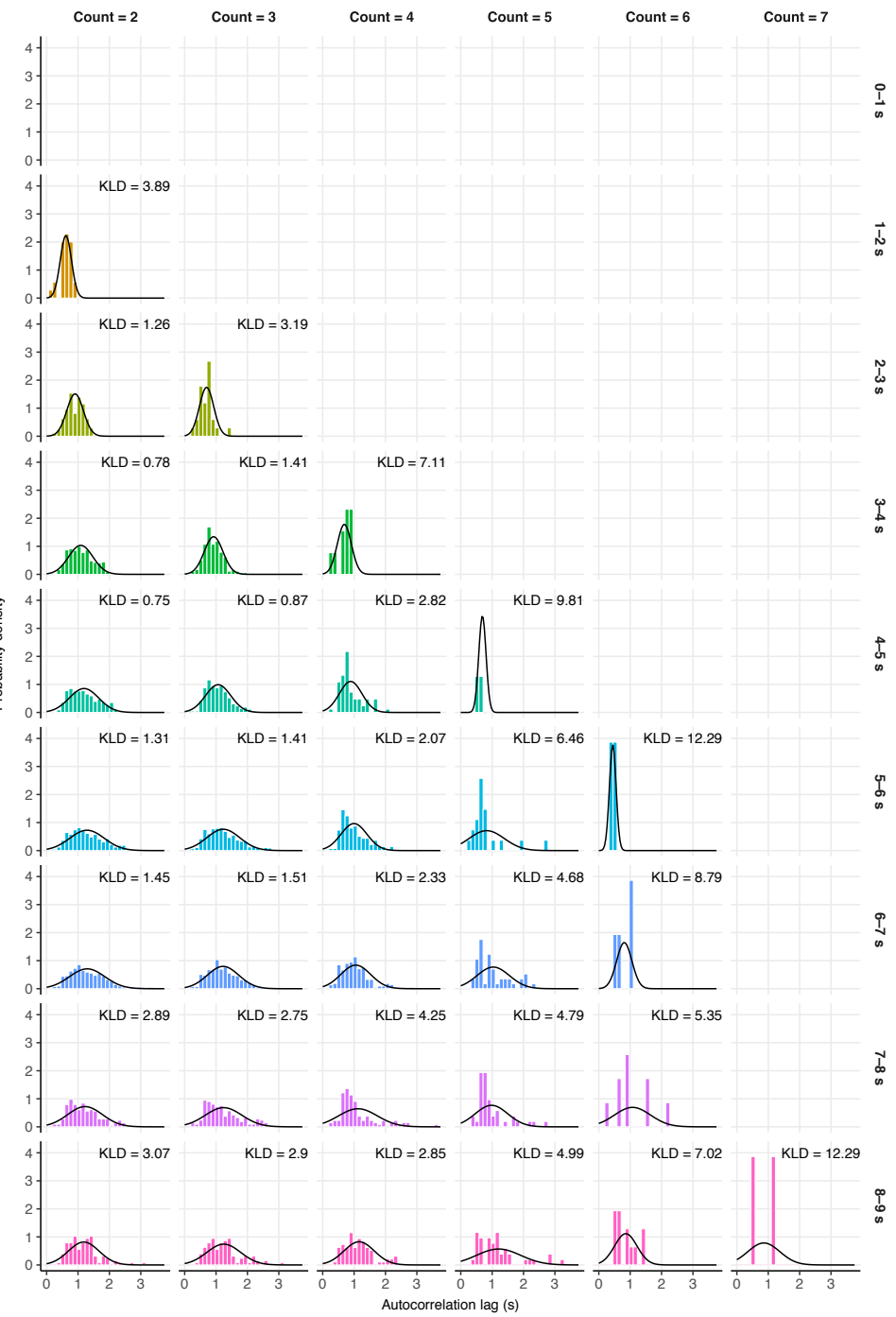

Figure 2: Periodicity of ips. (A) Horizontal boxplots of ip offsets, binned into 1-second IP duration bins (major ordinate); minor ordinate: ip index. (B) Probability density of autocorrelation lags (minor abscissa) as function of count of subsequent ip (major abscissa) and bin (major ordinate); black lines: normal probability density functions generated from mean and standard deviation of data; KLD = Kullback-Leibler Divergence.
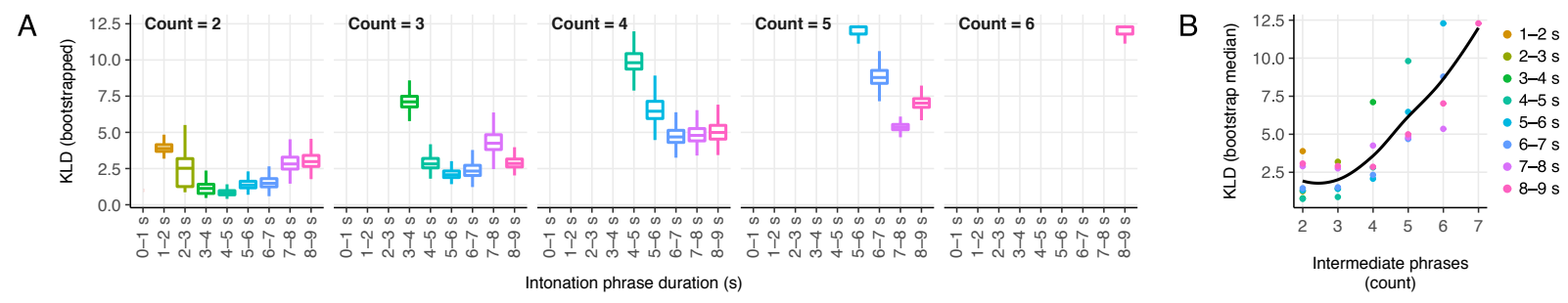

Figure 3: Dependence of ip periodicity on ip count within ip series. (A) Bootstrapped Kullback-Leibler Divergence (KLD) as function of number of successive ips (major abscissa) and IP duration bin; ordinate: KLD. (B) Correlation of median KLD with number of subsequent intermediate phrases, partialing out bin. 


\begin{tabular}{lcccccc}
\hline & \multicolumn{5}{c}{ Count of ips in series } \\
\cline { 2 - 7 } IP bin (s) & 2 & 3 & 4 & 5 & 6 & 7 \\
\hline $1-2$ & 610 & & & & & \\
$2-3$ & 930 & 730 & & & & \\
$3-4$ & 1,050 & 860 & 760 & 735 & & \\
$4-5$ & 1,140 & 1,000 & 785 & 650 & 445 & \\
$5-6$ & 1,180 & 1,140 & 900 & 650 & \\
$6-7$ & 1,200 & 1,165 & 990 & 885 & 830 & \\
$7-8$ & 1,140 & 1,100 & 935 & 795 & 930 & \\
$8-9$ & 1,140 & 1,170 & 1,090 & 1,040 & 750 & 860 \\
\hline
\end{tabular}

Table 2: Median autocorrelation lags per IP duration bin and count of ips within ip series, converted from samples to milliseconds.

\section{Discussion}

We here report that series of two to five subordinate ips are periodic within their superordinate IP. Second, the exact period of these series depends on the duration of the superordinate IP, with longer IPs containing also longer ips. This entails short-term periodicity of prosodic phrasing within a specific range.

Median autocorrelation lags ranged between 610 and 1,200 milliseconds, translating to a frequency between 1.64 and 0.83 Hertz (Table 2B). This is further evidence for an association between acoustic patterns in speech and periodic electrophysiological sampling time windows of the auditory system. Neuroscientific research has observed that oscillations below four Hertz synchronize with amplitude and frequency modulations of the low-passfiltered speech envelope (Bourguignon et al., n.d.; Meyer et al., 2016; Gross et al., 2013; Mai et al., 2016; Meyer, 2018). While we studied a symbolic annotation of ips instead of their acoustics, ip boundaries are defined by multi-dimensional modulations, second in ToBI break index strength only to IP boundaries (Silverman et al., 1992; Pierrehumbert, 1980). In line with this, a subset of these cues, amplitude modulations at the boundaries of intonation units analogous to ips, are periodic at 1 Hertz (Inbar et al., 2020; Chafe, 1979; Du Bois, 1992). We extend this work here in two important ways. While Inbar et al. (2020) analyzed periodicity within fixed windows of two seconds starting from the onset of an intonation unit, the hierarchical annotation scheme of ToBI allowed us to use IPs as windows. This revealed that periodicity outlasts time windows of two seconds (i.e., two intonation units), continuing for up to five ips (Figure 2B). In turn, this showed that ip period exhibits a range rather than being fixed to a strict one Hertz. The dependence on IP duration could even explain the cross-linguistic variance in periodicity observed by Inbar et al. (2020), which should be tested by future work.

But why does periodicity also not continue beyond five ips? Given that ip duration depends on IP duration (Figure 1), physiological constraints on IP production could explain 
this pattern (Vollrath et al., 1992). IP production requires exhalation, the frequency of which fluctuates with metabolic demands (Tort, Brankačk, \& Draguhn, 2018). IP offsets are defined by pitch and amplitude modulations as well as pauses, which accompany the end of a breathing cycle. Yet, only some pauses and pitch modulations at IP offsets coincide with the end of a breathing cycle (Grosjean et al., 1979; White, 2017; Pierrehumbert, 1979). Hence, cognitive constraints are a more plausible explanation: Information held in verbal working memory deteriorates over time, requiring timely higher-level integration of information across entire IPs (Christiansen \& Chater, 2015; Chafe, 1979; Simpson \& del Prado Martín, 2015; Speer et al., 1993; Christiansen \& Chater, 2015). While it has long been thought that the rate of deterioration depends on a constant decay function (Fuster, 1999; Scott et al., 2014), it has been argued that the specific contents of working memory, in particular the similarity of memorized items, affect the deterioration interval (Bays, 2015; van Dyke \& Johns, 2012; Oberauer \& Lange, 2008). The fact that deterioration becomes critical only beyond intervals of two seconds (Wingfield \& Byrnes, 1972; White, 2017; Miller, 1956; Cowan, 2001; Baddeley et al., 1975) fits the non-uniform distribution of IP duration that we observed. For speech, a memory interval of six words has been proposed, translating to roughly 2.4 seconds at average speech rates (Frazier \& Fodor, 1978; Tauroza \& Allison, 1990). A similar value of 2.7 seconds has been observed in electrophysiological studies (Hwang \& Steinhauer, 2011; Roll et al., 2012; Webman-Shafran et al., 2015; Schremm et al., 2015; Bögels et al., 2011).

One curious aspect of our results is the discrepancy between the median duration of ips across the corpus (i.e., 1.65 seconds) and the median periods of ip series (i.e., $610-1,200 \mathrm{~ms}$; Figures $1 \mathrm{~B} / \mathrm{C}$ and 3 and Table 2). The reason is the fact that ip series are autocorrelated only when successive ips have similar durations (e.g., short ips interspersed with a long ip do not yield autocorrelation). Furthermore, because shorter ips are more frequent than longer ips (i.e., the histogram in Figure 1A is skewed), autocorrelated series of long ips are less probable. In principle, this could mean that only short ips are periodic. An alternative interpretation follows the proposal that periodic electrophysiological sampling can tolerate and even compensate for aperiodic speech signals (Giraud, 2020; Meyer et al., 2020, 2019): If sampling is intrinsically periodic, sparse temporal series of ip boundaries might be enough for electrophysiology to stay in sync with speech. In line with this, oscillations at frequencies below four Hertz have not only been linked to overt prosody, but to the active inference of phrase boundaries when these are not acoustically marked (Meyer et al., 2016). Yet, a simple alternative explanation could also be the ambiguity of acoustic cues (Beckman, 1996) - intervening ip boundaries can easily be misclassified as pitch accents, full IP boundaries, or even missed by human annotators (Syrdal \& McGory, 2000; Grice et al., 1996, 2000). Importantly, any of these hypotheses could, in principle, also explain the apparent higher-order ip duration harmonics (Figure 1C). 
A difference to previous studies is the use of annotations instead of acoustics. Inbar et al. (2020) assessed the amplitude envelope, a critical cue for boundary perception (Brugos et al., 2018). It has been argued that the envelope dominates the cochlear output, which is handed to the cortex for the cognitive inference of boundaries (Ghitza et al., 2013; cf. Obleser et al., 2012). Yet, boundaries are a multi-dimensional construct (Brugos et al., 2018) and automatic detection of boundaries based on one acoustic dimension alone is inferior to multi-dimensional detection (Rosenberg, 2009). ToBI's formal definition of boundaries takes multi-dimensionality into account (Silverman et al., 1992) and human annotators tend to agree on the location of prosodic boundaries (Breen et al., 2012; Pitrelli et al., 1994). In principle, one could argue that human annotations do not reflect acoustic measurements alone, but are biased by perceptual and cognitive factors of the annotator. Still, we consider it unlikely that annotation labor as such induces periodicity of ips into a corpus. Nevertheless, future work should specify the link between the periodicity of the multiple dimensions of prosody and the periodicity of boundaries as such.

In order to generalize our results, the analyses should be repeated in corpora of further languages, registers, and annotation systems. First, there is likely cross-linguistic variability in periodicity, in particular when typological boundaries are transversed (Inbar et al., 2020). Second, idiosyncrasies of radio news prosody (Bolinger, 1982, 1989; Cotter, 1993) could lead to an overestimation of periodicity; hence, conversational and spontaneous speech should be assessed, which are characterized by short utterances, frequent turntaking, and many dysfluencies such as hesitations, pauses and speech repairs (Heeman \& Allen, 1999). Finally, the annotation inventory of the ToBI system could have biased the current analyses (Wightman, 2002), which should thus be replicated on corpora annotated with other standards (e.g., Inbar et al., 2020; Chafe, 1979; Du Bois, 1992).

\section{Acknowledgements}

This work was supported by the award of the Max Planck Research Group Language Cycles to Lars Meyer. We thank Dafydd Gibbon and Sascha Griffiths for helpful comments.

\section{References}

Arvaniti, A. (2012). The usefulness of metrics in the quantification of speech rhythm. Journal of Phonetics, 40(3), 351-373.

Baddeley, A. D., Thomson, N., \& Buchanan, M. (1975). Word length and the structure of short-term memory. Journal of Verbal Learning and Verbal Behavior, 14(6), $575-589$. 
Barr, D. J., Levy, R., Scheepers, C., \& Tily, H. J. (2013). Random effects structure for confirmatory hypothesis testing: Keep it maximal. Journal of Memory and Language, 68(3), 255-278.

Bates, D., Maechler, M., Bolker, B., \& Walker, S. (2015). Fitting linear mixed-effects models using lme4. Journal of Statistical Software, 67(1), 1-48. (doi:10.18637/jss.v067.i01)

Bays, P. M. (2015). Spikes not slots: Noise in neural populations limits working memory. Trends in Cognitive Sciences, 19(8), 431-438.

Beckman, M. E. (1996). The parsing of prosody. Language and Cognitive Processes, 11(1-2), 17-68.

Björkelund, A., Eckart, K., Riester, A., Schauffler, N., \& Schweitzer, K. (2014). The extended DIRNDL corpus as a resource for automatic coreference and bridging resolution. In In proceedings of LREC (pp. 3222-3228).

Bögels, S., Schriefers, H., Vonk, W., \& Chwilla, D. J. (2011). Prosodic breaks in sentence processing investigated by event-related potentials. Linguistics and Language Compass, 5(7), 424-440.

Bolinger, D. (1982). The network tone of voice. Journal of broadcasting, 26, 7726-728.

Bolinger, D. (1989). Intonation and its uses.

Bourguignon, M., De Tiege, X., de Beeck, M. O., Ligot, N., Paquier, P., Van Bogaert, P., ... Jousmäki, V. (n.d.). The pace of prosodic phrasing couples the listener's cortex to the reader's voice. Human Brain Mapping, 34.

Bower, G. H., \& Springston, F. (1970). Pauses as Recoding Points in Letter Series. Experimental Psychology, 83(3), 421-430.

Breen, M., Dilley, L. C., Kraemer, J., \& Gibson, E. (2012). Inter-transcriber reliability for two systems of prosodic annotation: ToBI (Tones and Break Indices) and RaP (Rhythm and Pitch).

Brugos, A., Breen, M., Veilleux, N., Barnes, J., \& Shattuck-Hufnagel, S. (2018).

Cue-based annotation and analysis of prosodic boundary events. In Proceedings of speech prosody (pp. 245-249).

Buzsáki, G. (2006). Rhythms of the Brain. New York.

Buzsáki, G. (2019). The Brain from Inside Out.

Chafe, W. L. (1979). The flow of thought and the flow of language. In Discourse and Syntax (pp. 159-181). Brill.

Christiansen, M. H., \& Chater, N. (2015). The Now-or-Never bottleneck: A fundamental constraint on language. Behavioral and Brain Sciences, 39, 1-72.

Cotter, C. (1993). Prosodic aspects of the broadcast news register. In Proceedings of the 19th Annual Meeting of the Berkeley Linguistics Society: General Session and Parasession on Semantic Typology and Semantic Universals (pp. 90-100). 
Coupé, C., Oh, Y., Dediu, D., \& Pellegrino, F. (2019). Different languages, similar encoding efficiency: Comparable information rates across the human communicative niche. Science Advances, 5(9), eaaw2594.

Cowan, N. (2001). The magical number 4 in short-term memory: A reconsideration of mental storage capacity. Behavioral and Brain Sciences, 24(1), 87-114.

Cummins, F. (2012). Oscillators and syllables: A cautionary note. Frontiers in Psychology, 3(OCT), 1-2.

Cutler, A., Dahan, D., \& Van Donselaar, W. (1997). Prosody in the Comprehension of Spoken Language: A Literature Review.

Ding, N., Patel, A. D., Chen, L., Butler, H., Luo, C., \& Poeppel, D. (2017). Temporal modulations in speech and music. Neuroscience and biobehavioral reviews, 81(Pt B), 181-187.

Doelling, K. B., Arnal, L. H., Ghitza, O., \& Poeppel, D. (2014). Acoustic landmarks drive delta-theta oscillations to enable speech comprehension by facilitating perceptual parsing. NeuroImage, 85, 761-768.

Du Bois, J. W. (1992). Discourse transcription. Santa Barbara papers in linguistics, 4, $1-225$.

Eckart, K., Riester, A., \& Schweitzer, K. (2012). A Discourse Information Radio News Database for Linguistic Analysis. In C. Chiarcos, S. Nordhoff, \& S. Hellmann (Eds.), Linked Data in Linguistics. Representing and Connecting Language Data and Language Metadata (pp. 65-75).

Farrell, S., \& Lelièvre, A. (2012). The dynamics of access to groups in working memory. Journal of Experimental Psychology: Learning Memory and Cognition, 38(6), 1659-1674.

Féry, C. (2017). Intonation and prosodic structure.

Frazier, L., Carlson, K., \& Clifton, C. (2006). Prosodic phrasing is central to language comprehension. Trends in Cognitive Sciences, 10(6), 244-249.

Frazier, L., \& Fodor, J. D. (1978). The sausage machine: A new two-stage parsing model. Cognition, 6(4), 291-325.

Fuster, J. M. (1999). Memory in the cerebral cortex: An empirical approach to neural networks in the human and nonhuman primate. Cambridge.

Ghitza, O. (2017). Acoustic-driven delta rhythms as prosodic markers. Language, Cognition and Neuroscience, 32(5), 545-561.

Ghitza, O., Giraud, A.-L. L., Poeppel, D., Miller, L. M., \& Davis, U. C. (2013). Neuronal oscillations and speech perception: Critical-band temporal envelopes are the essence. Frontiers in Human Neuroscience, 6(JAN), 1-4.

Gibbon, D. (2018). The Future of Prosody: It's about Time. In Proceedings of Speech Prosody (pp. 1-9). 
Giraud, A.-L. (2020). Oscillations for all A commentary on Meyer, Sun \& Martin (2020). Language, Cognition and Neuroscience, 1-8.

Giraud, A.-L., \& Poeppel, D. (2012). Cortical oscillations and speech processing: emerging computational principles and operations. Nature Neuroscience, 15(4), $511-7$.

Goswami, U., \& Leong, V. (2013). Speech rhythm and temporal structure: Converging perspectives? Laboratory Phonology, 4 (1), 67-92.

Grice, M., Ladd, D. R., \& Arvaniti, A. (2000). On the place of phrase accents in intonational phonology. Phonology, 143-185.

Grice, M., Reyelt, M., Benzmüller, R., Mayer, J., \& Batliner, A. (1996). Consistency in transcription and labelling of german intonation with gtobi. In Proceeding of the Fourth International Conference on Spoken Language Processing. ICSLP'96 (Vol. 3, pp. 1716-1719).

Grosjean, F., Grosjean, L., \& Lane, H. (1979). The patterns of silence: Performance structures in sentence production. Cognitive Psychology.

Gross, J., Hoogenboom, N., Thut, G., Schyns, P., Panzeri, S., Belin, P., \& Garrod, S. (2013). Speech rhythms and multiplexed oscillatory sensory coding in the human brain. PLoS Biology, 11(12), e1001752.

Heeman, P. A., \& Allen, J. F. (1999). Speech repairs, intonational phrases, and discourse markers: Modeling speakers' utterances in spoken dialogue. Computational Linguistics, 25(4), 527-571.

Henry, M. J., \& Obleser, J. (2012). Frequency modulation entrains slow neural oscillations and optimizes human listening behavior. Proceedings of the National Academy of Sciences, 109 (49), 20095-20100.

Hickok, G., Farahbod, H., \& Saberi, K. (2015). The rhythm of perception: entrainment to acoustic rhythms induces subsequent perceptual oscillation. Psychological science, 26(7), 1006-1013.

Howard, M. F., \& Poeppel, D. (2012). The neuromagnetic response to spoken sentences: Co-modulation of theta band amplitude and phase. NeuroImage, 60(4), 2118-2127.

Hwang, H., \& Steinhauer, K. (2011). Phrase length matters: The interplay between implicit prosody and syntax in Korean "garden path" sentences. Journal of Cognitive Neuroscience, $23(11)$, 3555-3575.

Inbar, M., Grossman, E., \& Landau, A. (2020). Sequences of intonation units form a 1 Hz rhythm. Sci Rep., Sep 28;10(1):15846.

Jun, S. A. (2010). Prosodic Typology. In Prosodic typology: The phonology of intonation and phrasing.

Kelso, J. A. S., Saltzman, E. L., \& Tuller, B. (1986). The dynamical perspective on speech production: Data and theory. Journal of Phonetics, 14(1), 29-59. 
Kreiner, H., \& Eviatar, Z. (2014). The missing link in the embodiment of syntax: Prosody. Brain and Language, 137, 91-102.

Ladd, D. R. (2008). Intonational phonology. Cambridge University Press.

Lehiste, I. I., Olive, J. P., Streeter, L. A., \& Lehiste, I. I. (1976). Role of duration in disambiguating syntactically ambiguous sentences. The Journal of the Acoustical Society of America, 60(5), 1199-1202.

Luo, H., \& Poeppel, D. (2007). Phase patterns of neuronal responses reliably discriminate speech in human auditory cortex. Neuron, 54 (6), 1001-1010.

Mai, G., Minett, J. W., \& Wang, W. S. (2016). Delta, theta, beta, and gamma brain oscillations index levels of auditory sentence processing. NeuroImage, 133, $516-528$.

Maris, E., \& Oostenveld, R. (2007). Nonparametric statistical testing of EEG- and MEG-data. Journal of Neuroscience Methods, 164(1), 177-190.

Mayer, J. (1995). Transcribing German intonation - the Stuttgart system (Tech. Rep.). University of Stuttgart.

Meyer, L. (2018). The neural oscillations of speech processing and language comprehension: state of the art and emerging mechanisms. European Journal of Neuroscience, 48(7), 2609-2621.

Meyer, L., Henry, M. J., Gaston, P., Schmuck, N., \& Friederici, A. D. (2016). Linguistic Bias Modulates Interpretation of Speech via Neural Delta-Band Oscillations. Cerebral Cortex, 27(9), 4293-4302.

Meyer, L., Sun, Y., \& Martin, A. E. A. (2019). Synchronous, but not entrained: exogenous and endogenous cortical rhythms of speech and language processing. Language, Cognition and Neuroscience, 0(0), 1-11.

Meyer, L., Sun, Y., \& Martin, A. E. A. (2020). "Entraining" to Speech, Generating Language? Language, Cognition and Neuroscience, 0(0), 1-11.

Miller, G. A. (1956). The magical number seven, plus or minus two: some limits on our capacity for processing information. Psychological Review, 63(2), 81-97.

Nespor, M., \& Vogel, I. (2007). Prosodic phonology: with a new foreword (Vol. 28). Walter de Gruyter.

Oberauer, K., \& Lange, E. B. (2008). Interference in verbal working memory: Distinguishing similarity-based confusion, feature overwriting, and feature migration. Journal of Memory and Language, 58, 730-745.

Obleser, J., Herrmann, B., \& Henry, M. J. (2012). Neural oscillations in speech: Don’t be enslaved by the envelope. Frontiers in Human Neuroscience, 6(AUGUST), 2008-2011.

Peelle, J. E., Gross, J., \& Davis, M. H. (2013). Phase-locked responses to speech in human auditory cortex are enhanced during comprehension. Cerebral Cortex, $23(6), 1378-1387$. 
Pierrehumbert, J. (1979). The perception of fundamental frequency declination. The journal of the acoustical society of America, 66(2), 363-369.

Pierrehumbert, J. (1980). The phonology and phonetics of english intonation (Unpublished doctoral dissertation). Massachusetts Institute of Technology, Dept. of Linguistics and Philosophy.

Pitrelli, J., Beckman, M., \& Hirschberg, J. (1994). Evaluation of prosodic transcription labeling reliability in the ToBI framework. In Proceedings of the 3rd workshop on spoken language processing (pp. 123-126).

Price, P. J., Ostendorf, M., \& Park, M. (2015). The use of prosody in syntactic disambiguation and syntactic structure are neither are of spoken With few exceptions. Journal of Acoustic Society of America, 90 (December 1991), $2296-2970$.

R Core Team. (2017). R: A language and environment for statistical computing [Computer software manual]. Vienna, Austria.

Roll, M., Lindgren, M., Alter, K., \& Horne, M. (2012). Time-driven effects on parsing during reading. Brain and Language, 121(3), 267-272.

Rosenberg, A. (2009). Automatic detection and classification of prosodic events. Columbia University.

Schremm, A., Horne, M., \& Roll, M. (2015). Brain responses to syntax constrained by time-driven implicit prosodic phrases. Journal of Neurolinguistics, 35, 68-84.

Schroeder, C. E., \& Lakatos, P. (2009). Low-frequency neuronal oscillations as instruments of sensory selection. Trends in Neurosciences, 32(1), 9-18.

Scott, B. H., Mishkin, M., \& Yin, P. (2014). Neural correlates of auditory short-term memory in rostral superior temporal cortex. Current Biology, 24(23), 2767-2775.

Selkirk, E. (1984). Phonology and syntax: The relation between sound andstructure. Cambridge, MA: MIT Press. Selkirk1984Phonology and syntax: The relation ....

Silverman, K., Beckman, M., Pitrelli, J., Ostendorf, M., Wightman, C., Price, P., ... Hirschberg, J. (1992). ToBI: A standard for labelling English prosody. In Proceedings of the 2nd international conference on spoken language processing (pp. 867-870).

Simpson, H., \& del Prado Martín, F. M. (2015). Memory capacity limits in processing of natural connected speech: The psychological reality of intonation units. In 37th annual conference of the cognitive science society, pasadena, ca. (pp. 2206-2211).

Snedeker, J., \& Casserly, E. (2010). Is it all relative? Effects of prosodic boundaries on the comprehension and production of attachment ambiguities. Language and Cognitive Processes, 25(7-9), 1234-1264.

Speer, S. R., Crowder, R. G., \& Thomas, L. M. (1993). Prosodic Structure and Sentence Recognition. Journal of Memory and Language. 
Speer, S. R., \& Ito, K. (2009). Prosody in first language acquisition - Acquiring intonation as a tool to organize information in conversation. Linguistics and Language Compass.

Steinhauer, K., Alter, K., \& Friederici, A. D. (1999). Brain potentials indicate immediate use of prosodic cues in natural speech processing. Nature Neuroscience, 2(2), 191-196.

Sturges, P. T., \& Martin, J. G. (1974). Rhythmic structure in auditory temporal pattern perception and immediate memory. Journal of Experimental Psychology, 102(3), 377-383.

Syrdal, A. K., \& McGory, J. (2000). Inter-transcriber reliability of ToBI prosodic labeling. 6th International Conference on Spoken Language Processing, ICSLP 2000 (Icslp).

Szymanski, F. D., Rabinowitz, N. C., Magri, C., Panzeri, S., \& Schnupp, J. W. (2011). The laminar and temporal structure of stimulus information in the phase of field potentials of auditory cortex. Journal of Neuroscience, 31(44), 15787-15801.

Tauroza, S., \& Allison, D. (1990). Speech rates in British English. Applied Linguistics, $11(1), 90-105$.

Tilsen, S., \& Arvaniti, A. (2013). Speech rhythm analysis with decomposition of the amplitude envelope: Characterizing rhythmic patterns within and across languages. The Journal of the Acoustical Society of America, 134, 628-.

Tort, A. B., Brankačk, J., \& Draguhn, A. (2018). Respiration-Entrained Brain Rhythms Are Global but Often Overlooked (Vol. 41) (No. 4).

Truckenbrodt, H. (1999). On the relation between syntactic phrases and phonological phrases. Linguistic Inquiry, 30(2), 219-255.

van Dyke, J. A., \& Johns, C. L. (2012). Memory Interference as a Determinant of Language Comprehension. Linguistics and Language Compass, 6(4), 193-211.

Vollrath, M., Kazenwadel, J., \& Krüger, H.-P. P. (1992). A universal constant in temporal segmentation of human speech. Naturwissenschaften, 79(10), 479-480.

Wagner, M., \& Watson, D. G. (2010). Experimental and theoretical advances in prosody: A review. Language and Cognitive Processes.

Wahl, A. (2015). Intonation unit boundaries and the storage of bigrams. Review of Cognitive Linguistics, 13(1), 191-219.

Webman-Shafran, R., Fodor, J. D., Janet, R. W.-s., \& Fodor, D. (2015). Phrase Length and Prosody in On-Line Ambiguity Resolution. Journal of Psycholinguistic Research, 45(3), 447-474.

White, P. A. (2017). The three-second "subjective present": A critical review and a new proposal. Psychological Bulletin, 143(7), 735-756.

Wightman, C. W. (2002). ToBI or not ToBI? In Proceedings of speech prosody (pp. 25-29). 
Wightman, C. W., Shattuck-Hufnagel, S., Ostendorf, M., \& Price, P. J. (1992).

Segmental Durations In The Vicinity Of Prosodic Phrase Boundaries. Journal of the Acoustical Society of America, 91 (3), 1707-1717.

Wingfield, A., \& Byrnes, D. L. (1972). Decay of information in Short-term memory. Science, 176 (4035), 690-692. 